\title{
Quality Control of Herbal Material and Phytopharmaceuticals with MS and NMR Based Metabolic Fingerprinting
}

Authors

Affiliation
Frank van der Kooy, Federica Maltese, Young Hae Choi, Hye Kyong Kim, Robert Verpoorte

Division of Pharmacognosy, Section of Metabolomics, Institute of Biology, Leiden University, Leiden, The Netherlands
Key words

- metabolic fingerprinting

- NMR

- MS

- medicinal plants

- herbal plant preparations

\begin{abstract}
$\nabla$

Metabolic fingerprinting techniques have received a lot of attention in recent years and the annual amount of publications in this field has increased significantly over the past decade. This increase in publications is due to improvements in the analytical performance, most notably in the field of NMR and MS analysis, and the increased awareness of the different applications of this growing field. Metabolomic fingerprinting or profiling is continuously being applied to new
\end{abstract}

\section{Introduction}

$\nabla$

Plants have been used throughout history as the primary source of food, timber, fuel, and medicine, among other purposes. Due to their amazing ability to produce a vast structural array of chemical components, of which only relatively few have been identified, plants are one of the most important sources for the development of new drugs. Today plants, plant extracts and plant-derived components are being used in a multitude of herbal remedies and phytopharmaceuticals. Well-known plants which are used to treat specific diseases include Artemisia annua (to treat uncomplicated malaria) [1] and the female flowering tops of Cannabis sativa (to relieve pain and as an appetite enhancer) [2]. A multitude of examples exists for the use of plant extracts to treat a large array of diseases and the WHO estimates that about $80 \%$ of the world population uses medicinal plants as their primary source of medication. Many well-known drugs have also been isolated from plants including artemisinin from A. annua, paclitaxel from Taxus brevifolia, vinblastine and vincristine from Catharanthus roseus and galanthamine from the Amaryllidaceae family. In the literature three terms are used to describe the analysis of metabolites produced by plants. areas of research such as drug discovery from natural resources, quality control of herbal material, and discovering lead compounds. In this review the current state of the art of metabolic fingerprinting, focussing on NMR and MS technologies will be discussed. The application of these two analytical tools in the quality control of herbal material and phytopharmaceuticals forms the major part of this review. Finally we will look at the future developments and perspectives of these two technologies in the quality control of herbal material.
These terms, notably metabolic profiling, metabolic fingerprinting and metabolomics are sometimes used interchangeably. It is not our intention to give a formal definition of metabolomics. For a formal definition and discussion $[3,4]$ should be consulted. A very simplistic view is that metabolomics can be seen in two different ways. Firstly there is the microscopic view (targeted approach) which looks at a specific set of compounds (e.g., phenolics or terpenoids, etc). This approach is also called metabolic fingerprinting or metabolic profiling. The second view is the macroscopic view (untargeted approach) which aims at identifying and quantifying all the metabolites present in a specific organism [3]. Metabolomics is therefore a "systematic study of the unique chemical fingerprints that specific cellular processes leave behind" - and, more specifically, the study of their small-molecule metabolite profiles [5]. The metabolome represents the collection of all metabolites in a biological organism, which are the end products of its gene expression. Thus, while mRNA gene expression data and proteomic analyses do not tell the whole story of what might be happening in a cell, metabolic fingerprinting can give an instantaneous snapshot of the physiology of that cell. One of the challenges of systems biology is to integrate proteomic, transcriptomic, and 
metabolomic information to give a more complete picture of living organisms.

The analytical tools used to study the metabolome include mainly chromatography and spectroscopy. Chromatography is used to separate the metabolites which are then being quantified and identified with the use of various detectors. The two main chromatography systems employed in metabolic fingerprinting are gas chromatography (GC) and high-performance liquid chromatography (HPLC). The detectors used in these chromatography systems depend largely on the type of components to be detected and quantified. For a targeted approach, mass spectroscopy (MS) is usually employed as the detector, while nuclear magnetic resonance (NMR) and MS are used without the chromatography step as an untargeted analytical tool. In the 1980s the first reports on the use of NMR for fingerprinting of plant extracts were reported, e.g., Kubeczka and Formácek published a book [6] with ${ }^{13} \mathrm{C}$-NMR fingerprints of essential oils and Schripsema and Verpoorte applied ${ }^{1} \mathrm{H}-\mathrm{NMR}$ for characterizing plant cell cultures and quantitative analysis of sugars in the cell culture extracts [7]. But, particularly in the past decade, metabolomics is rapidly developing for studying plants and quality control of botanicals. The use of these two analytical tools (MS and NMR) will be discussed in more detail below as well as the applications in the quality control of herbal material and the perspectives of these methods.

\section{Metabolic Fingerprinting using NMR}

Recent advances in analytical chemistry, combined with multivariate data analysis, have brought us closer to the final goal of metabolomics, comprehensive evaluation of all metabolites, both quantitatively and qualitatively in living organisms. Among many different technological platforms, NMR and MS have been successfully used for metabolic fingerprinting analysis. These two techniques have their respective advantages and limitations, and are often discussed as being complementary [8,9]. However, as a tool for metabolomics, NMR has some unique advantages over MS-based methods. It can provide a detailed analysis on the biomoleculear composition very quickly with relatively simple sample preparation [10-12]. It is a universal detector for all mole- cules containing NMR-active nuclei, unlike MS where detection of analytes is influenced by selective ionization or ultraviolet spectrometers where only chromophore-bearing compounds are detected. For all proton-bearing molecules, the intensity of all proton signals is absolutely proportional to the molar concentration of the metabolite. Using a proper internal standard, the real concentration of metabolites can be easily calculated [11]. Because NMR spectroscopy is based on the physical characteristics of compounds, it has a very high reproducibility.

In metabolomics, the detection of metabolites is not the final step, but all data obtained from the analytical methods should be further analyzed by statistical methods in order to extract all possible information from the data. The accuracy and correctness of the data to be further analyzed by the statistical methods are inevitably reliant on the robustness of the raw analytical data set. In this aspect, NMR has a unique advantage, the highest reliability in metabolomics. Unlike the retention time in chromatography-based techniques, with a few exceptions the chemical shift, coupling constant, and integral of each signal in an NMR spectrum does not change as long as it is measured under the same conditions: the field strength applied, solvent, $\mathrm{pH}$, and the temperature. Despite the low intrinsic sensitivity, the robustness of data and ability to cover a broad range of metabolites, has enabled NMR to be the favored overall "macroscopic" metabolomics and fingerprinting tool. In addition to the advantages of data robustness, the power of NMR in structure elucidation of metabolites cannot be matched by any other method. NMR has a long history in the natural products chemistry field as the tool for structural elucidation. With a proper database, it can generate data which can be kept almost permanently [13]. In this part, the general procedure of NMR metabolomic analysis will be discussed together with multivariate data analysis. The identification of metabolites is of high importance and this aspect will be highlighted. At the end of this section specific applications will be discussed using NMR as a metabolic fingerprinting technique.

\section{General procedures of NMR-based metabolomics}

NMR-based metabolomics, like other methods using MS or chromatography, include sample preparation, extraction, multivariate data analysis, and identification of metabolites. $\bullet$ Fig. 1 shows

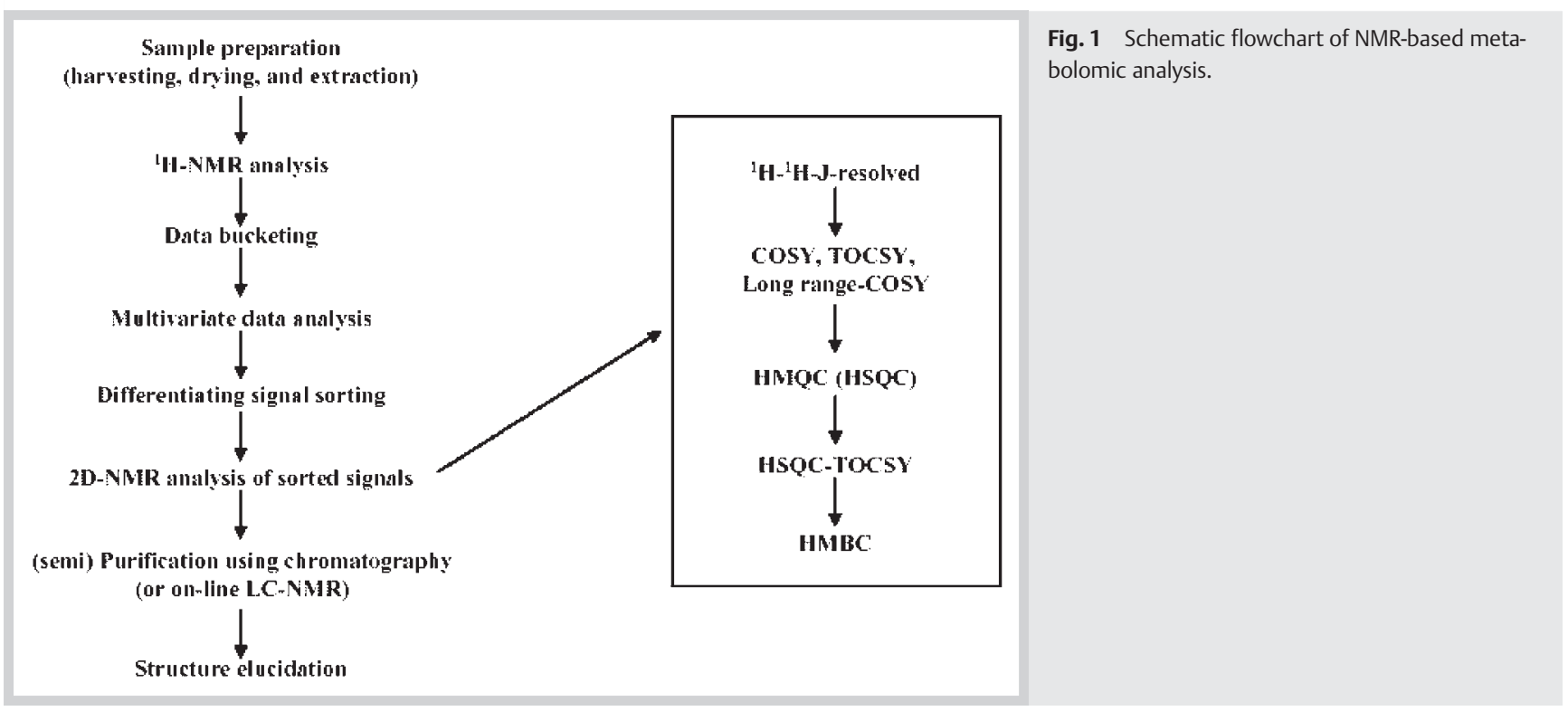


the generally accepted procedure of NMR-based plant metabolomics. The sample preparation steps need much caution in order to obtain reproducible and reliable results. Because in the metabolomic analysis, the age, type of tissues, developmental stage, environmental conditions (e.g., water drought, sunlight, temperature, and $\mathrm{pH}$ of soil), and harvesting time greatly affect the metabolome obtained even from the same genotype. For these aspects sampling of the target materials should be carefully planned to avoid increased biological variation interfering with the final data interpretation [10]. After harvesting, the sample should be frozen immediately to avoid any (bio)chemical change in the material. The next step is grinding and extraction of the material to liberate the metabolites from the cells. During the extraction (bio)chemical reactions may occur in the material, reflected in changes in the metabolome. This can be avoided by drying the material before extraction either by heat or freezedrying, keeping the material at a low temperature, or grinding at low temperatures and/or in the presence of a solvent that denatures enzymes involved in metabolite alteration. Denaturation can also be achieved by a brief microwave treatment. For the development of an optimum extraction method, Ultra-Turrax ${ }^{\circledR}$, deep-freezing in liquid nitrogen followed by mechanical grinding, sonication with a probe head or cup horn and bead beating prior to solvent extraction were evaluated for the efficient breakage of the cell using a mycobacteria, Mycobacterium bovis. Of the evaluated methods sonication was found to be superior to others [14].

As metabolomics aims at comprehensive fingerprinting of all metabolites the extraction methods used should cover all possible metabolites in an organism. Unfortunately, there is no single extraction method to achieve that goal. The polarity of the solvent limits the range of metabolites that can be extracted. Also the $\mathrm{pH}$ is a factor that affects the profile of metabolites extracted, e.g., alkaloids are soluble in non-polar solvents at high $\mathrm{pH}$, and in aqueous solvents at low $\mathrm{pH}$. To overcome these problems in part, a two-phase solvent system consisting of chloroform-methanolwater $(2: 1: 1)$ has been applied to extract both polar and nonpolar compounds in a single extraction [15-18]. Also, this method was applied to metabolic fingerprinting of Ephedra with a slight modification, by addition of $\mathrm{NH}_{4} \mathrm{OH}$, for benzylamine alkaloids extraction [19]. However, this elaborate two-phase extraction method was found to be problematic when dealing with a large number of samples because of the long processing time and the possibility of degradation or losses during processing. Recently, a simple direct extraction method with deuterated NMR solvents has been developed for sample preparation, which in general gave better quality NMR spectra [20-24].

Single solvent systems have been used for some studies. Methanol with TFA was found to be a suitable solvent for the extraction of alkaloids [25] while perchloric acid was routinely used for the more polar metabolites, which prevents enzymatic degradation of metabolites [26]. However, a combination of $\mathrm{CD}_{3} \mathrm{OD}$ and $\mathrm{D}_{2} \mathrm{O}$ was found to be a more preferable solvent, since it can extract more diverse metabolites. The combination of these two solvents was used in different ratios, e.g., $30 \%$ to $70 \%$ of $\mathrm{CD}_{3} \mathrm{OD}$ depending on the study $[20,23,24]$. In most of our work, a mixture of methanol- $d_{4}$ and $\mathrm{KH}_{2} \mathrm{PO}_{4}$ buffer in $\mathrm{D}_{2} \mathrm{O}$ (pH 6.0) has been used, which extracts a wide range of metabolites including amino acids, carbohydrates, fatty acids, organic acids, phenolics, and terpenoids in a single step [19-22]. The direct extraction method with deuterated NMR solvents can save time and make it possible to deal with a large number of samples. Moreover, the buffer in the sol- vent can avoid possible fluctuation of chemical shifts of signals in the NMR spectra.

However, when commercial herbal preparations are analyzed a more targeted approach is used focussing on the known major bioactive compounds in the plant material. For instance, in the case of St John's wort (Hypericum perforatum), both tablets and capsules were analyzed using a mixture of $\mathrm{MeOH}$-pyridine $(6: 4$, v/v) [27].

\section{${ }^{1} \mathrm{H}-\mathrm{NMR}$ analysis to detect all metabolites extracted from samples}

For NMR-based metabolomics, the ${ }^{1} \mathrm{H}-\mathrm{NMR}$ spectrum provides a wealth of chemical information. With the aid of chemical shifts and coupling constants some metabolites (amino acids, phenolics, sugars, and TCA-related organic acids) can be easily identified. The observed chemical shift positions and spin-spin coupling pattern for each proton provide information as to what kinds of protons are found in the molecules and subsequently how the protons are arranged. Furthermore, the concentration of each metabolite in the sample can be easily calculated from the integration of the signals in the spectra. There is no requirement for calibration curves to convert signal intensity into concentration as used in other methods $[8,28-30]$. For obtaining reproducible results on concentration, the following parameters are important: relaxation delay, the pulse width, and the acquisition time. The optimum range of these parameters in ${ }^{1} \mathrm{H}-\mathrm{NMR}$ was excellently reviewed by Pauli et al. [9]. One of the problems in measuring ${ }^{1} \mathrm{H}-\mathrm{NMR}$ spectra is the water signal, a huge signal caused by residual water, which overlaps with the anomeric protons of sugars or glycosides $(\delta=4.8-5.2)$. To suppress this undesired water signal several methods have been applied, e.g., addition of paramagnetic ions like $\mathrm{Mg}^{2+}$ followed by Meiboom-Gill modification of the Carr-Purcell (CPMG) spin-echo pulse program [31] and pre-saturation using an additional pulse. When the pre-saturation technique is applied during relaxation delay, the most common method, unwanted reduction of the signal intensity close to the suppressed water might occur. For correct suppression, the temperature and $\mathrm{pH}$ of samples should be carefully adjusted since the water signal is greatly affected by those factors. Depending on the molecular size of metabolites, specific pulse sequences have been applied. To filter out the signals from small molecules from those of large ones, spin diffusion differences can be utilized. This was nicely shown in an NMR-based study on toxin-induced changes in lipoprotein profiles [32]. In the case of a matrix containing macromolecules (e.g., proteins or lipid vesicles) the application of a spin-echo sequence like the CPMG pulse sequence allows the attenuation of unwanted resonances from macromolecules [33]. However, in any ${ }^{1} \mathrm{H}-\mathrm{NMR}$ spectra of plant extracts using diverse pulse sequences there are congested signals of metabolites. Multivariate data analysis is a key step for sorting out the discriminating signals from the complex spectra. Prior to the multivariate data analysis all signals in the obtained ${ }^{1} \mathrm{H}-\mathrm{NMR}$ spectra should be digitalized. This could be achieved by the so-called bucketing procedure, which divides the spectrum into small bins and sums all intensities in each bin. Ideally, a smaller bucket size is better to examine subtle perturbations in the metabolome. However, because of unfavorable fluctuations of chemical shifts, even with a fixed temperature and $\mathrm{pH}$ of samples, one can only use $8-16 \mathrm{~Hz}$ (0.02 to $0.04 \mathrm{ppm}$ in $400 \mathrm{MHz}$ NMR) for the bucket size [34]. It is also possible to use full-resolution NMR data for the multivariate data analysis as shown in the study of Rasmussen et al. [27]. In this work, they 
showed that full-resolution NMR data sets $(0.15 \mathrm{~Hz}$ per data point resulting in 30000 variables) gave more precise information about constituents responsible for data clustering, compared to the use of integrated NMR data sets $(0.04$ ppm bucketing resulting in 200 variables). Some aligning methods were found to improve the separation of NMR data for further statistical analysis [35-37].

\section{Multivariate data analysis of ${ }^{1} \mathrm{H}-\mathrm{NMR}$ spectra to} recognize patterns and find discriminating signals In recent metabolomics studies, many hundreds or sometimes thousands of samples are routinely analyzed and a minimum of several hundreds of signals are detected in each sample. It is crucial to extract the relevant information from such a huge data set. The samples are grouped based on the analysis of the metabolome (pattern recognition) and a discriminating metabolite (biomarker) can be found by multivariate data analysis. The pattern of a class represents the information about the relations between the observations within the class, discerning which are similar, which are different, or which are atypical outliers. Through the pattern recognition some metabolites can be found that are characteristic of a class. Of the multivariate data analysis, principal component analysis (PCA) and partial least squares projections to latent structures (PLS) are on the verge of becoming routine processing steps for raw analytical data. PCA is designed to extract and display the systematic variation in a data matrix and PLS is a regression extension of PCA, which is used to reveal the correlation between two kinds of data sets [38-41]. Both PCA and PLS are projection methods by reducing original data to a few principal components in order to describe maximum variation within the data. All raw data (e.g., the integral values of all chemical shifts in a ${ }^{1} \mathrm{H}-\mathrm{NMR}$ spectra) of samples are plotted in a K-dimensional space ( $K=$ number of variables, e.g., chemical shifts in ${ }^{1} \mathrm{H}-\mathrm{NMR}$ spectra) and the plotted samples are projected onto the line generated by least square sense (principal component line). The score of each sample is obtained along the PC line. The next principal components can also be calculated by projection onto the line which is orthogonal to the previous PC line or space. In general, the first three PCs are used for the analysis. For the data analysis, score and loading plots are used. The score plot is useful for observation of any groupings in the data set and in addition will highlight outliers that may be due to errors in sample preparation, experimental conditions or instrumentation parameters. Coefficients by which the original variables should be multiplied to obtain the PC are called loadings. The numerical value of a loading of each variable on a PC shows how much the variable has in common with that component. Prior to the PCA and PLS analysis raw data are scaled in different ways. There are several ways to scale the data. For unit-variance scaling, the standard deviation and the scaling weight as the inverse standard deviation are calculated. Subsequently, each variable is multiplied by the inverse standard deviation. Each scaled variable then has equal variance. This method is useful when the classification relies more on differences between minor compounds, since it increases the influence of weaker signals. However, applying the unit-variance scaling to an NMR data set, it is difficult to interpret the loading plot obtained from the unit-variance scaling method because all signals have the same variance and consequently are different from the original NMR spectra. With mean-centering, the average value of each variable is calculated and then subtracted from the data. This improves the interpretability of the loading plot since it resembles the original NMR spectra. Also, the spectral noise is removed for the analysis as long as intensities of noise are lower than real signals. However, the influence of minor signals might be excluded since the mean-centering method always retains the original value of signals. In particular, the signals of plant secondary metabolites can be underestimated compared to high levels of primary metabolites. As an alternative technique the so called Pareto scaling has become more common. Pareto scaling gives each variable a variance numerically equal to its initial standard deviation instead of unit-variance. Hence, Pareto scaling is intermediate between the extremes of mean-centered and unit-variance scaling [42], which gives some weight to minor signals and at the same time it provides interpretable loadings. In recent years variable stability (VAST) scaling which weights each variable according to a metric of its stability improved the class distinction and predictive power of PLS-DA models for ${ }^{1} \mathrm{H}-\mathrm{NMR}$ spectra of urines [43].

\section{Two-dimensional NMR spectroscopy to identify} differentiating metabolites in the NMR spectra

The signals to discriminate sample groups are sorted by multivariate data analysis. Some metabolites like carbohydrates, amino acids, or organic acids related to the TCA cycle can be elucidated merely based on their chemical shifts in ${ }^{1} \mathrm{H}-\mathrm{NMR}$ spectra. However, for most cases, the spectral complexity and signal overlap in the ${ }^{1} \mathrm{H}-\mathrm{NMR}$ spectra are too high for identification. Chemical structures of metabolites, especially species-specific plant secondary metabolites, should be confirmed by additional methods. Diverse two-dimensional (2D) NMR spectroscopy can give information on chemical structures. Indeed one of the advantages of NMR spectroscopy in plant metabolomics is the power of structure elucidation of molecules in a complex mixture.

The 2D-NMR spectroscopy available for metabolomics can be divided into two types, resolved and correlated spectroscopy according to the type of dispersion in the second frequency dimension [8]. A typical resolved technique is the $2 \mathrm{D}-{ }^{1} \mathrm{H}-{ }^{1} \mathrm{H}-J$-resolved spectra. It provides additional information on spin-spin coupling constants, together with chemical shifts. One of the difficulties for interpreting a ${ }^{1} \mathrm{H}-\mathrm{NMR}$ spectrum is the interpretation of the splitting pattern of each signal. If a target signal is detected in a crowded region overlapping with other signals, the first step is to identify its splitting pattern. In addition to this use of $J$-resolved spectra in structure elucidation, projected spectra on to the axis of chemical shift have been applied in several studies $[20-22,44]$. The projected spectrum produces a ${ }^{1} \mathrm{H}-\mathrm{NMR}$ spectrum consisting of singlets for each ${ }^{1} \mathrm{H}$ chemical shift, i.e., a fully proton-decoupled proton NMR spectrum. In the projected spectrum all signals from macromolecules which have short $\mathrm{T}_{2}$ relaxation times are readily suppressed because the pulse sequence of the $J$-resolved technique is based on a spin echo sequence [8]. Thus, baseline shifting caused by proteins, oligo- or polysaccharides, which might overlap minor signals or result in overestimation of target signals, are clearly reduced in the projected spectra and lead to better separation in multivariate data analysis. Another promising resolved technique is diffusion-ordered spectroscopy (DOSY) where each NMR signal is resolved based on the molecular diffusion coefficient [45]. DOSY can be used for molecular weight evaluation of unknown, purified constituents by preparing calibration curves with known compounds [46]. Although DOSY has a potential to differentiate metabolites having the same chemical moieties and different molecular weights in plant extract, e.g., glycosides from their aglycones, the application in metabolomics has been limited so far to the analysis of 
polymers like polysaccharides from insects or mushrooms [47, $48]$, interaction between small molecules and ligands $[49,50]$, and hydrocarbon mixtures [51].

Following the resolved spectra, information on correlation between protons or carbon-proton can be obtained by homonuclear and heteronclear experiments. A typical proton homonuclear technique is correlation spectroscopy (COSY) that shows which signals in a proton spectrum have mutual spin-spin couplings. The resulting spectrum has the conventional 1D NMR spectrum along the diagonal cross-peaks at chemical shifts corresponding to pairs of coupled nuclei. In general protons within three bonds are well correlated in the COSY spectrum but in $s p^{2}$ spin systems such as olefinic and phenolic compounds, even the correlation beyond three bonds is readily detected. A modified pulse sequence of COSY, long range COSY, can selectively detect correlation between protons far from each other (more than three bonds). It can show structure confirmation of connectivity between two molecules which is not possible to detect in a normal COSY spectrum.

It is one of the most time-consuming tasks to define NMR signals belonging to the same molecule in the complex ${ }^{1} \mathrm{H}-\mathrm{NMR}$ spectra of a mixture. In particular, most ${ }^{1} \mathrm{H}$ signals of carbohydrates are detected in an extremely crowded region $(\delta=3.0-4.5)$ overlapping with each other or with the signals of amino acid protons close to the amino group. A powerful 2D-NMR technique, total correlation spectroscopy (TOCSY) or the Hartmann-Hahn experiment (HAHAHA) provides information on unbroken chains of coupled protons in the same molecule. For example, if anomeric protons of carbohydrates are defined, the rest of the signals in the crowded region can be easily assigned using the correlation in the TOCSY spectrum.

All organic molecules have carbons as their chemical backbone. The information on the carbons is thus crucial for structure elucidation. In fact measurement of a ${ }^{13} \mathrm{C}-\mathrm{NMR}$ spectrum is an important, if not indispensable, step to elucidate the chemical structure of a pure compound. However, the magnetically active ${ }^{13} \mathrm{C}$ isotope has too low a sensitivity for the application to the analysis of the metabolome since the natural abundance of ${ }^{13} \mathrm{C}$ is only $1.1 \%$ of ${ }^{12} \mathrm{C}$ and its magnetogyric ratio one fourth of ${ }^{1} \mathrm{H}$. It results in $1 / 57000$ of the ${ }^{1} \mathrm{H}$ sensitivity. Also, the long relaxation time of ${ }^{13} \mathrm{C}$ requires a long measuring time in order to obtain quantitative results. To overcome the low sensitivity of ${ }^{13} \mathrm{C}$ heteronuclear correlation techniques are used from which the information on ${ }^{13} \mathrm{C}$ chemical shifts is indirectly obtained.

Direct correlation between carbons and protons can be detected by heteronuclear multiple quantum coherence (HMQC) or heteronuclear single quantum coherence (HSQC) which gives better sensitivity than HMQC but needs phase correction. In both HMQC and HSQC spectra any carbon attached directly to a proton is detected, consequently the chemical shift of a ${ }^{13} \mathrm{C}$ connected to a specific proton can be obtained. The assignment of C-6 and C-8 of 5,7-dihydroxyflavonoids can easily be learned from the HMQC spectra of the extract of aerial parts of Genista tenera. In the HMQC spectra the unusual upfield-shift of those aromatic carbons compared to other aromatic carbons, caused by the adjacent oxygen, confirmed the presence of 5,7-dihydroxyflavonoids [52]. Long-range correlations (two and three bonds) between carbons and protons can be observed by heteronuclear multiple bond correlation (HMBC) spectra. It is an extremely powerful tool for structure elucidation since carbon-carbon correlations can be indirectly obtained with the aid of HMQC or HSQC. In addition, correlations between quaternary carbons like carboxylic acid and nearby protons can be seen in the spectra. The long-range couplings in HMBC spectra can give information on the connectivity between two moieties which are connected only by quaternary carbons or hetero atomes (e.g., $\mathrm{O}$-glycosides). One of the advantages of NMR-based metabolomics is that there are numerous combinations of 2D-NMR experiments that provide information on structures by diverse correlations. Aiming at specific correlations, enhancing resolution, or simplifying spectra HMBC-J-resolved [39], DOSY-selective TOCSY [53], 2D-J-DOSY [54] and DOSY-HMQC [55] have been applied to the analysis of complex mixtures. The combined HMQC (or HSQC)-TOCSY sequence experiment leads to a ${ }^{13} \mathrm{C}$-edited TOCSY spectrum, and information on all connectivities of carbons in each molecule in a mixture.

\section{Application to Plant Products}

\section{$\nabla$}

\section{Classification}

NMR-based metabolic fingerprinting has been applied to diverse fields of plant research. Classification and identification of adulteration of plant products are among the major interests. Using NMR-based metabolic analysis, it was possible to discriminate different species of plants. An example is the study of Ilex [17]. Eleven species of Ilex were analyzed and based on their metabolites, each species could be discriminated, especially Yerba mate (I. paraguariensis) from its adulterants. The contributing metabolites were arbutin, phenylpropanoids, caffeine and theobromine. Caffeine and theobromine were only found in the I. paraguariensis, whereas arbutin was found only in other species. The study of flower heads of chamomile (Matricaria recutita) showed that this technique can be applied to assess the relative amount of stalk materials in the different batches of flowers [56]. Another example of NMR analysis combined with PCA is the application to characterize 3 different Strychnos species using different parts of the plants (seeds, roots, leaves and barks). All samples were clearly classified based on their metabolites such as brucine, loganin, Strychnos icaja alkaloids (icajine, sungucine) as well as fatty acids [57].

Twelve Cannabis sativa cultivars were discriminated by analyzing the organic fractions and aqueous fractions. THCA and CBDA were the most important metabolites in organic fractions, while primary metabolites found in the water fraction such as glucose, asparagine and glutamic acid served as discriminating metabolites [15].

\section{Quality control in traditional Chinese medicines}

Traditional Chinese medicines (TCM) are often used as a mixture of several plants, with a hardly defined composition. Even though there are regulations for quality control, this is often restricted only to a certain compound or group of compounds. NMR-based metabolic fingerprinting combined with pattern recognition may be a very good tool for the quality control of TCM. Chemotaxonomic analysis including quality control issues was applied in the study of Ephedra [19]. Ephedra is one of the oldest medicinal plants known to mankind. However, 3 different species of Ephedra, E. sinica, E. intermedia, and E. equisetina are commonly and widely used without strict distinction as long as there is a certain amount of ephedrine alkaloids ( $0.8 \%$ of dry weight). Using NMRbased metabolic fingerprinting, it was possible to discriminate $E$. sinica, E. intermedia and E. distachya. Not only the ephedrine type of alkaloids, but also another secondary metabolite (benzoic acid analogue) was found to be an important discriminating metabo- 

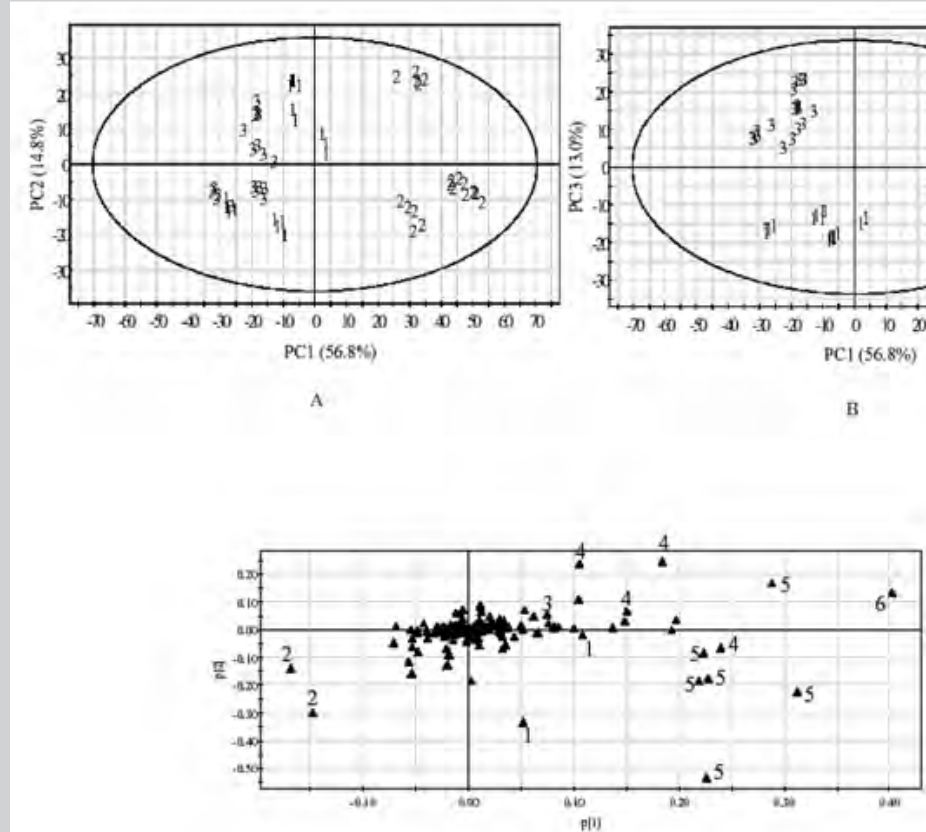

C

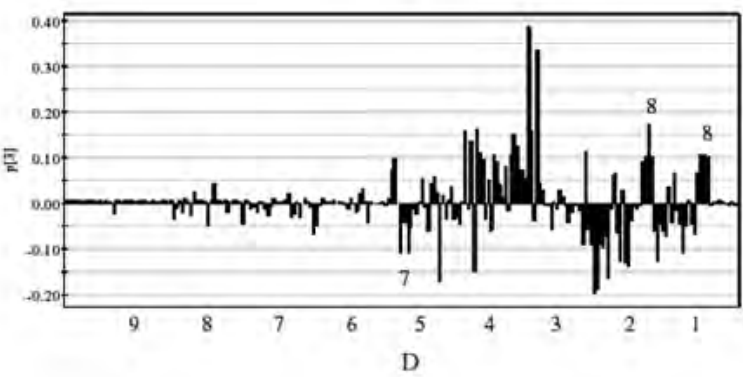

Fig. 2 Score plot of principal component analysis of ginseng preparations using PC1 vs. PC2 (A) and PC1 vs. PC3 (B). 1-3; commercial ginseng preparations. And loading plot of principal component analysis of ginseng preparations. PC1 and PC2 (C) and PC3 (D). 1, alanine; $\mathbf{2}$, arginine; $\mathbf{3}$, fumaric acid; 4, inositol; $\mathbf{5}$, sucrose; $\mathbf{6}$, acetate; $\mathbf{7}$, anomeric protons of sugars; $\mathbf{8}$, methyl protons of saponins (adapted from [58] reproduced with permission of Georg Thieme Verlag KG Stuttgart, New York). lite. Among nine tested commercial Ephedra materials, one was shown to be the mixture of two species. There are several reports on the discrimination of ginseng (Panax ginseng) preparations, which is one of the most popular herbal medicines. The first report [58] using NMR-based metabolomics approach showed the differences in ginseng products, different ages ( 4,5 and 6 years old) and differently processed (white and red) ( Fig. 2). PCA of $J$-resolved ${ }^{1} \mathrm{H}$-NMR spectra of three commercial ginseng preparations showed striking differences among the preparations (- Fig. 2A and B). Loading plots explained that alanine, arginine, fumaric acid, inositol and ginsenosides are important metabolites to differentiate preparations from each other ( Fig. $2 \mathrm{C}$ and D). Other studies also showed that this approach can be used for the quality control of fresh ginseng roots of different ages [59] and different origins [60]. Recently, another study for the quality evaluation of TCM was reported by Tarachiwin and coworkers [61]. A combination of an NMR technique and multivariate analysis showed the differences in two Angelica acutiloba roots, A. acutiloba Kitagawa (yamato-toki) and A. acutiloba Kitagawa var. sukiyamae Hikino (hokkai-toki), with regard to their geographical and variety origin.

Application of NMR to commercial preparations of Artemisia has been reported recently by Van der Kooy et al. [62]. NMR analysis combined with PCA showed clear differences between $A$. annua and $A$. afra. A more targeted analysis combined with LC-MS showed that the content of artemisinin in the commercial prod- ucts is different from the commercial claim. Other studies of NMR-based methods were applied to the characterization of crude extracts and commercial preparations, including feverfew [63], Kava-kava [64], Artemisia [65] and St. John's wort [66]. The details were reviewed in a recent review by Holmes [67].

\section{MS-Based Metabolomics \\ $\nabla$}

Hyphenated chromatography-MS systems employed for metabolomic fingerprinting consist of three parts. The first part is the chromatographic separation section, used to separate complex mixtures obtained from the plant material. The most commonly used chromatographic systems are HPLC, GC or CE. The second part consists of the ionization chamber were the molecules are being ionized. Different ionization sources exist such as electron ionisation (EI), electrospray ionization (ESI), atmosperic pressure chemical ionization (APCI) and atmospheric pressure photoionization (APPI). Another ionization source being used, but not in combination with flow-based systems, is matrix-assisted laser desorption/ionization (MALDI). The third part consists of the MS were the ionized molecules are being detected as their mass-tocharge ratio. The most commonly used MS systems include single quadrupole, triple quadrupole, ion trap and time-of-flight spectrometers. More advanced high resolution spectrometers include the Orbitrap or the Fourier transform-cyclotron. For an in depth 


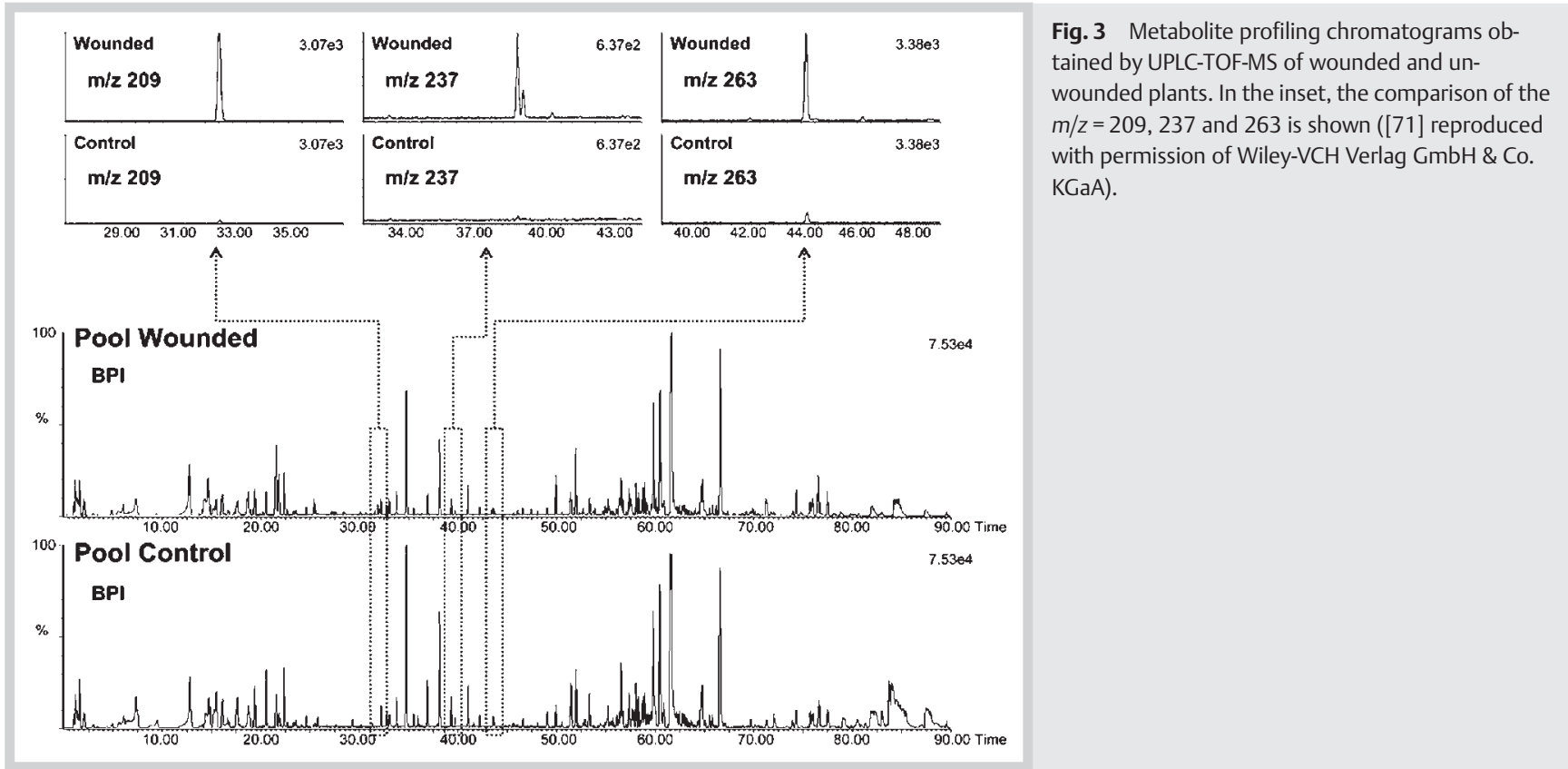

review of ionization sources and mass spectrometers reference [68] should be consulted. There is little difference in the sample preparation and data handling between MS-based and NMRbased technologies, although the solvents being used differ between the two analytical systems. NMR makes use of deteurated solvents while MS uses non-deteurated solvents.

\section{Sample preparation and extraction}

Various authors have made use of targeted extraction methods in order to extract and analyze a specific group of components. Garcia-Villalba et al. [69] used various solvents to extract a major number of compounds from soybean seeds in a comparative study between transgenic and conventional soybeans. The solvents tested for their extraction efficiency included: aqueous methanol (60, 80, 90 and $100 \%, v / v)$, aqueous ethanol $(60,80,90$ and $100 \%, v / v$ ), ethyl acetate and aqueous acetonitrile (70 and $100 \%, v / v)$. An alternative extraction procedure was also tested by removing the fat (mainly phospholipids) with hexane prior to the extraction. They concluded that $80 \%$ methanol resulted in the highest number of metabolites extracted. In a study to compare the phenolic content between the leaves, stems, roots and fruits of Vaccinium angustifolium, 95\% ethanol was used as extraction solvent [70]. Grata et al. [71] used $50 \mathrm{mg}$ of lyophilized leaf tissues and extraction with isopropyl alcohol using a ball mill in a case study of the wound response in Arabidopsis thaliana. - Fig. 3 illustrates the UPLC-TOF-MS chromatograms obtained from the wouded plants in comparison to the control plants. Vorst et al. [72] studied a non-directed unbiased total extraction of metabolites from tubers (Solanum tuberosum). The material was ground to a fine powder in liquid nitrogen. Extracts of $0.5 \mathrm{~g}$ frozen powder with $2 \mathrm{~mL} 62.5 \%$ methanol, $0.125 \%$ formic acid in water at $0{ }^{\circ} \mathrm{C}$ were prepared in duplicate. After immediate mixing, the extracts were sonicated for $10 \mathrm{~min}$, spun down $(10 \mathrm{~min}$ at $1000 \mathrm{~g}$ ) and filtered through a $0.2 \mu \mathrm{m}$ inorganic filter. Recently another extraction protocol for a untargeted metabolomic approach for plant metabolomics was published [73], based on reversed-phase liquid chromatography coupled to high-resolution mass spectrometry (LC-QTOF MS) of aqueous methanol extracts.
It can be concluded that no single extraction solvent will be able to extract all metabolites present. This limitation holds unfortunately true for all analytical procedures including NMR-based metabolite fingerprinting.

\section{Sample analysis}

The most important limitations of MS-based methods are the inherent lack of ionization of certain components. Ionized components can be identified and quantified at very low quantities making the MS a very sensitive analytical tool for metabolic fingerprinting. The lack of ionization and therefore the inability to quantify or identify components that do not ionize limits the use of MS to the more targeted metabolic profiling techniques. Various probes are being employed to overcome this inherent ionization problem in order to increase the dynamic range of components that can be detected with the use of MS. Other limitations of MS-based technologies are that the equipment needs to be calibrated regularly and fragmentation of molecules occurs which complicates the mass spectra. Fragmentation does however lead to structural information which in turn leads to identification of unknown components with the use of MS-MS-based technologies.

Samples prepared for MS analysis are usually separated chromatographically before the separated compounds are analyzed with the MS. Separation technologies employed largely make use of GC $[74,75]$, HPLC $[70,76]$, and capillary electrophoresis (CE) [69, $77,78]$. These technologies are either employed to obtain a targeted chemical fingerprint of a specific plant extract or as an untargeted technique in order to quantify and identify as many metabolites as possible in a plant tissue.

APCI and ESI are the most frequently used with HPLC chromatography as the separation step. These probes are known as "soft ionization" probes, while the detector used with these probes is usually the ion trap or the single quadrupole detector $[70,79$ 81]. EI ionization, also known as "hard ionization", is more frequently employed with GC as the chromatography step. More advanced MS technologies and also the more expensive technolo- 
gies employed to conduct metabolic fingerprinting include the FT Cyclotron MS [82,83] and time of flight MS [76,78].

\section{Data handling}

Due to the inherent variation and reproducibility problems associated with any chromatography technique, the generated data should be aligned before any data reduction techniques such as PCA or PLS can be employed. Alignments are used to reduce the variation in retention times between samples. Dedicated software, such as MetAlign, is used for the alignment of chromatographic peaks, automated baseline correction and alignment of all mass peaks across all samples. Subsequent statistics and bioinformatic tools can be used to provide a detailed view on the differences and similarities between samples or to link metabolomics data to other systems biological information [73]. During another study and with the use of the software "Similarity Evaluation System for Chromatographic Fingerprint of TCM", nine common peaks were identified which were used to evaluate 13 batches of Baishi samples from different provinces in China [84]. Various other processing software systems are currently available which aim at reducing variation between sample sets and to extract as much information as possible from the raw data sets. GC-MS data were processed by [85] with MATLAB software 7.1 (Mathworks) while [86] used MET-IDEA to process data generated by GC, LC or CE chromatography in combination with MS systems.

\section{Applications of Chromatography-MS for Quality Control of TCM \\ $\checkmark$}

Chromatography-MS technologies are mainly employed in a targeted approach when the quality control of TCM products is involved. Kang et al. [84]. used an HPLC-PDA-ESI-MS for the chromatographic fingerprinting of furocoumarins in the roots of Angelica dahurica. A novel and sensitive HPLC-DAD-ESI/MS method has been developed by Su et al. [87], for the simultaneous determination of five major flavonoids in Hypericum japonicum hydroalcoholic extracts. Fingerprinting of TCM is also used to identify substances illegally mixed into TCM preparations. Liu et al. [88] identified aminophyline and prednisone acetate mixed illegally in TCM preparations. Li et al. [89] developed an approach with the use of HPLC/DAD and GC/MS in order to fingerprint aporphinoid and quinolizidine alkaloids of the total alkaloids from Caulophyllum robustum. The results were used to construct binary chromatographic fingerprints of the total alkaloids. Artemisia annua is a potent antimalarial herbal drug. This activity is ascribed to artemisinin, a sesquiterpene lactone that is very effective against drug-resistant Plasmodium species with a low toxicity. As a consequence, in view of a possible use of the phytocomplex rather than pure artemisinin, an HPLC/DAD/MS method was proposed for the simultaneous detection and quantification of both flavonoids and artemisinin. Jensen et al. [90] developed a LC-MS-APCI method in order to evaluate the composition of active constituents in phytopharmaceutical preparations. Several commercial ginkgo preparations on the Swiss market were analyzed, and the ginkgolide and bilobalide contents were evaluated. In another study a comparative analysis of the flavonoid components of the leaves of two medicinal plants known in Brazil as "espinheira santa", namely, Maytenus ilicifolia and M. aquifolium and a hybrid plant, M. aquifolium $\times$ M. ilicifolia, has been carried out using HPLC coupled with PDA-MS. By combining efficient LC

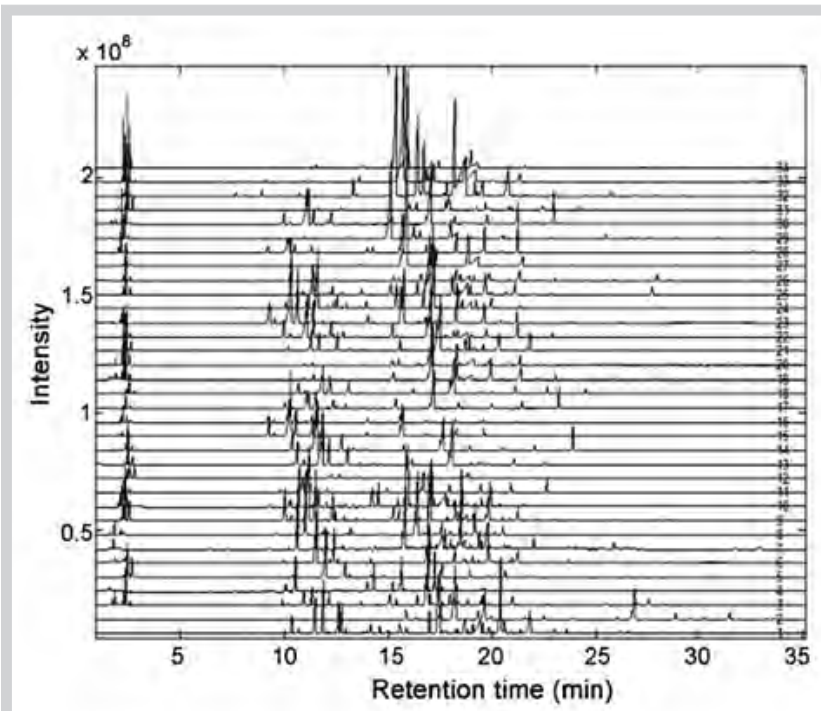

Fig. 4 The total ion current (TIC) chromatograms of 34 GC-MS data of fresh medicinal materials of Houttuynia cordata collected from four representative planting areas in China ([74] reproduced with permission of Elsevier B.V.).

separation and ESI-multiple stage mass spectrometry (ESI-MSn) with UV detection, performed both with and without the postcolumn addition of shift reagents, the on-line identification of minor flavonoids in Maytenus extracts could be readily achieved. Fingerprint analysis of the flavonoid extracts revealed significant differences in the profiles of the two Maytenus species, while the hybrid plant contained flavonoids found in both parent species [91]. Bruni et al. [92] examined the qualitative and quantitative phytochemical variations in the dried flowering tops of cultivated Hypericum perforatum infected by phytoplasmas of the "ash yellows" class. Methanolic extracts of healthy and infected plants were separated by RP-HPLC to quantify naphthodianthrones and flavonoids, while essential oils were analysed by means of GC/MS. The affected plants exhibited decreased amounts of rutin, hyperoside, isoquercitrin, amentoflavone and pseudohypericin whereas the chlorogenic acid content was doubled. The essential oil yield was drastically reduced in infected material and revealed an increased abundance of sesquiterpenes and a matching decrease in monoterpene hydrocarbons and aliphatics. The consequences of the phytopathological condition of cultivated $H$. perforatum plants on the commercial quality were further outlined. Zeng et al. [74] employed GC-MS to develop a quality control method on thirty-four samples of medicinal materials of Houttuynia cordata collected from four different areas. O Fig. 4 illustrates a comparison of the total ion chromatograms of the 34 samples analyzed by GC-MS.

\section{Trends}

$\nabla$

The greatest challenge that metabolomics studies face is metabolite differentiation and identification. Technical advances of current analytical tools are promising in this perspective. NMR is the tool traditionally utilized by natural products chemists for structure elucidation. The major limitation of NMR is its rather low sensitivity when compared to other analytical methods. Recently 
developed probes for high-resolution NMR, such as cryoprobes and capillary NMR, help to increase the sensitivity threshold of the technique. CryoNMR aims to limit the receiver coil noise and to improve the coil quality factor by cooling the receiver coil and the preamplifiers to $25 \mathrm{~K}$ or below. With the use of cryogenically cooled probes the detection sensitivity of NMR is at least 3- to 4fold enhanced in comparison with conventional probes operating at the same magnetic field [93]. Capillary NMR takes advantage from the use of small diameter radiofrequency (RF) coils for mass-limited samples (usually in the order of nanomole quantities). By decreasing the diameter of the receiver coil, the signalto-noise $(\mathrm{S} / \mathrm{N})$ ratio improves [94]. Both cryoNMR and capNMR are very promising analytical approaches for metabolic fingerprinting studies.

The importance of "chemically characterizing" the metabolic compounds has been pointed out by Kopka as one of the main goals for any further development of the metabolomics approach [95]. As no single analytical technique is suitable for the detection and identification of all the metabolites in a biological sample, the most common strategy to clean up or isolate and structurally identify metabolites is to combine chromatographic techniques to MS or/and NMR spectroscopy. The resulting hyphenated methods, such as various HPLC-NMR techniques, are succesfully applied to natural products research. An off-line solid phase extraction may sometimes be required to partially purify or concentrate the analytes [96,97]. Pioneering in the field of medicinal plants were Hostettmann, Wolfender and coworkers, who since the late 1990s have applied continuous and stopped-flow HPLC-NMR for the investigation of many natural products [98102], and many other publications as reviewed by Jaroszewski [103]. More recently Wolfender and coworkers developed hyphenated methods for non-targeted metabolome profiling of minor metabolites from plant extracts $[104,105]$. The strategy is based on ultra-performance liquid chromatography-time-offlight mass spectrometry (UPLC-TOF-MS). UPLC is a newly developed analytical technique that seems to be very promising in metabolic fingerprinting and metabolomics studies. The method is based on the use of very small particles $(1.7 \mu \mathrm{m})$ at elevated pressure, thus achieving superior theoretical plate numbers and resolution in a short time of analysis and with less solvent consumption $[106,107]$. When applied to natural products analysis, UPLC revealed great advantages in comparison to standard HPLC [108-110]. The combination of the technique with mass spectrometry has been applied for the analysis of biological fluids, achieving very high resolution and sensitivity with the concurrent advantage of reduced separation speed [111-114]. A first attempt at using UPLC-MS for the differentiation of complex herbal mixtures was done by Jacob et al. [115]. The author applied UPLC-MS in combination with chemometrics tools for a chromatographic fingerprinting of 24 different Chinese medicinal herbs. The aim was to detect toxic compounds (aristolochic acids) in the multiherbal preparations of the traditional Chinese medicine. MS is often chosen as detection method coupled to UPLC, but this requires a sample preparation (e.g., pretreatment with solid phase extraction or liquid-liquid extraction) that is considered the ratelimiting factor of the method [107].

Another advanced liquid chromatographic technique that has been applied only a few times in metabolic fingerprinting is the hydrophilic interaction chromatography (HILIC). The method utilizes hydrated silica columns and eluting systems that are moving from high organic solvents miscible with water to high aqueous solvents. Water forms stagnant water layers on the stationary

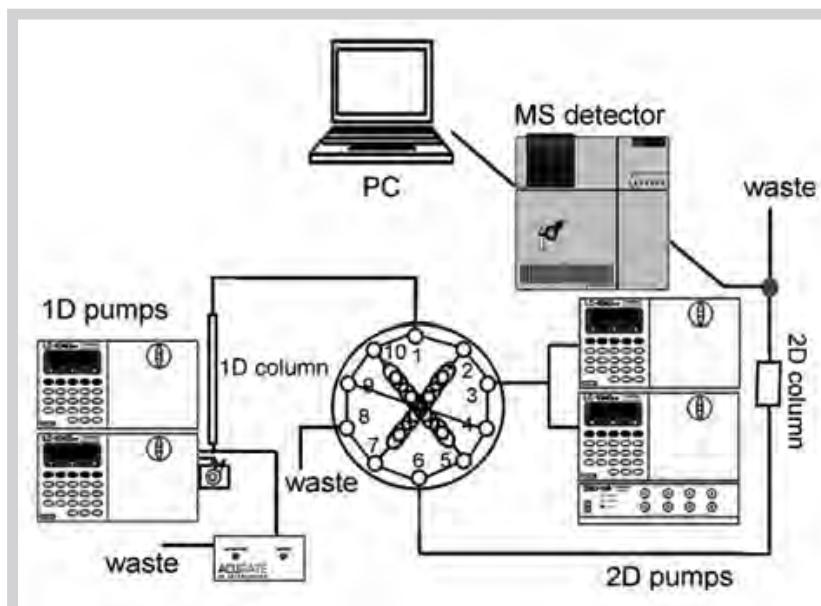

Fig. 5 Conventional $\mathrm{LC} \times \mathrm{LC}$ set-up using one multi-port switching valve between the two dimensions ([122] reproduced with permission of Elsevier B.V.).

phase surface and the polar compounds are partitioned in this system [116]. The method is applicable to the analysis of hydrophilic compounds that are subjected to high retention by the column. Tolstikov and Fiehn applied HILIC chromatography coupled to mass detectors for the analysis of plant extracts [117] and later for a comprehensive analysis of urine [118]. Oligosaccharides, glycosides, amino sugars, amino acids, and sugar nucleotides were detected in the phloem exudates from Cucurbita maxima leaves by the authors. In order to cover a wider metabolome range, Dunn proposed that HILIC chromatography should be connected with reverse-phase liquid chromatography [119]. Although the mentioned enhanced liquid chromatographic techniques have been developed, the peak resolution is still the limiting factor when LC is chosen for comprehensive metabolic profiling. The introduction of a second dimension has proved to be a powerful strategy although, up to now, it has been mainly applied in proteomics $[120,121]$. When multidimensional chromatography is applied, the sample is subjected to two analytical separations, which are columns with orthogonal properties based on different selectivities. A combination of 2D liquid chromatography $($ Ag-LC $\times$ RP-LC) and a mass spectrometric detection method (APCI-MS) was applied for a comprehensive analysis of triacylglycerols in commercial natural oil by Dugo and coworkers. 0 Fig. 5 illustrates a conventional LC $\times$ LC set-up which was used during the analysis [122]. The analytes were clustered in groups in the 2D space thus facilitating their classification, and they were identified on the basis of the APCI-mass spectra. However, the method still suffers of some limitations such as mobile phase immiscibility, precipitation of buffer salts and incompatibility of stationary phase [123].

Analogously to two-dimensional liquid chromatography, also gas chromatography has seen the development of GC $\times$ GC for the analysis of targeted and/or unknown volatile compounds present in a mixture. The separation efficiency is highly increased especially for very complex samples. Advantages are the fast acquisition time of the technique, and with the use of the second dimension the problem of co-elution is in most of the cases reduced. Compared to one-dimensional GC, the sensitivity of the chromatographic technique is much enhanced (trace levels of compounds are detected) and chemically similar compound patterns 
are created with a major advantage for the identification of unknown compounds [124]. The combination of the GC × GC system with a mass spectrometer represents a very comprehensive tool for the analysis of complex mixtures. Only a limited number of applications of this platform have been reported so far. The twodimensional gas chromatography technique coupled with quadrupole mass spectrometric detector (GC $\times$ GC-qMS) was employed by Shellie and coworkers for the metabolic differentiation of Pelargonium graveolens essential oils [125] and of semi-volatile oils from roots of three different ginseng species [126]. Very high efficiency was obtained, with mass spectra of high quality that facilitated the identification of separated metabolites by matching the results with available libraries. The same authors applied a GC $\times$ GC approach coupled to time-of-flight MS (GC $\times$ GC-TOF-MS) for a metabolomic study of mammalian tissues [127]. The TOF-MS detector has the advantage of an efficient spectral deconvolution of partially resolved GC $\times$ GC elutes that gives more structural information. In the study of Shellie and colleagues, the technique led to the detection of more than 1200 compounds, with a number of peaks characterized by high "analytical purity" that was increased by a factor of seven when compared to the classical one-dimensional GC-TOF method. The authors could structurally characterize only $10 \%$ of all the detected compounds. Nevertheless, the method showed to be effective for the determination of some metabolomic biomarkers of obese and lean mice. Hope and coworkers demonstrated the importance of using tandem GC in combination with a TOF mass spectrometer for the analysis of complex samples, such as metabolic extracts from perennial rye grass for an evaluation of the pre- and postharvest physiology of the samples [128]. This was a very earlystage study of GC $\times$ GC-TOF-MS applied to plants for the purpose of metabolomic studies. The results of the analysis were limited only to one single identified compound (malic acid) but the method showed to have advantages of enhanced resolution and increased speed when compared with 1D chromatography. In another metabolomic-based study of plants a combination of GC $\times$ GC-TOFMS and principal component analysis (PCA) was applied to chemically differentiate the extracts from basil, peppermint, and stevia. The regions of interest, as resulted from the PCA, were further deconvoluted by the use of the chemometric technique parallel factor analysis (PARAFAC) and the interesting analytes were quantified [129]. The GC $\times$ GC technique is a promising tool and it is expected to gain a much wider distribution.

However, analytical progresses will not lead to any improvement in the field of metabolomics science if we do not consider also other points of view. Metabolomics "happens" within each cell, and the tissues that are the object of our investigation at the moment are made of several cell types [130]. The next generation of plant metabolome scientists must focus on the cellular and even subcellular compartmentation of metabolism. Researchers have started looking into the metabolomics of single cells with the use of laser microdissection (LMD). In combination with GC-TOF-MS analysis the technique has been applied for the first time to a comprehensive metabolic fingerprinting of vascular bundles of Arabidopsis after the choice of cryosectioning the tissue as pretreatment procedure [131]. The study led to the identification of 68 metabolites. For more than half of them the authors could demonstrate whether they were enriched or depleted in vascular bundles. LMD has also been successfully used in combination with cryoprobe NMR for the analysis of specialized cells from the floral and leaves tissues of Dilatris spp. coming from different herbarium specimens [132]. The phytochemical pattern of
LMD-derived samples was compared with that of whole leaf extracts and some secondary metabolites were identified and connected with the specific cells. The study revealed the potential of the method in chemotaxonomic applications even of plants that are difficult to raise or to obtain. The same kind of approach, in combination with mass spectrometric measurements (LMD/ $\mathrm{NMR} / \mathrm{MS}$ ) was carried out on the stone cells of Norway spruce bark [133].

The next point that must be considered is that metabolism takes place as a network happening within very short time fractions (<msec), and not as individual pathways. It seems thus more realistic to describe the metabolome of a tissue (or a cell) not as a single snapshot, but measuring the actual metabolic flux.

\section{References}

1 Klayman DL. Qinghaosu (artemisinin): an antimalarial drug from China. Science 1985; 228: 1049-1055

2 Small E. Cannabis as a source of medicinals, nutraceuticals, and functional foods. In: Acharya SN, Thomas JE, editors. Advances in medicinal plant research. Kerala: Research Signpost; 2007: 1-39

3 Villas-Boas SG, Rasmussen S, Lane GA. Metabolomics or metabolite profiles? Trends Biotechnol 2005; 23: 385-386

4 Goodacre R, Vaidyanathan S, Dunn WB, Harrigan GG, Kell DB. Metabolomics by numbers: acquiring and understanding global metabolite data. Trends Biotechnol 2004; 22: 245-252

5 Daviss B. Growing pains for metabolomics. Scientist 2005; 19: 25-28

6 Kubeczka K.-H, Formácek V. Essential oils: analysis by capillary gas chromatography and carbon 13-NMR spectroscopy. London: John Wiley \& Sons Ltd.; 1980

7 Schripsema J, Verpoorte R. Investigation of extracts of plant cell cultures by proton nuclear magnetic resonances spectroscopy. Phytochem Anal 1991; 2: 155-162

8 Reily MD, Lindon JC. NMR spectroscopy: principles and instrumentation. In: Robertson DG, Lindon J, Nicholson JK, Holmes E, editors. Metabonomics in toxicity assessment. Boca Raton: CRC Press; 2005: 75104

9 Seger C, Sturm S. Analytical aspects of plant metabolic profiling platforms: current standings and future aims. J Proteome Res 2007; 6: 480-497

10 Choi YH, Kim HK, Verpoorte R. Metabolomics. In: Kayser O, Quax W, editors. Medicinal plant biotechnology from basic research to industrial applications. Weinheim: Wiley-VCH; 2006: 9-28

11 Pauli GF, Jaki BU, Lankin DC. Quantitative ${ }^{1} \mathrm{H}$ NMR: development and potential of a method for natural products analysis. J Nat Prod 2005; 68: $133-149$

12 Verpoorte R, Choi YH, Kim HK. NMR-based metabolomics at work in phytochemistry. Phytochem Rev 2007; 6: 3-14

13 Breitling R, Ritchie S, Goodenowe D, Stewart ML, Barrett MP. Ab initio prediction of metabolic networks using Fourier transform mass spectrometry data. Metabolomics 2006; 2: 155-164

14 Jaki BU, Franzblau SG, Cho SH, Pauli GF. Development of an extraction method for mycobacterial metabolome analysis. J Pharm Biomed Anal 2006; 41: 196-200

15 Choi YH, Kim HK, Hazekamp A, Erkelens C, Lefeber AWM, Verpoorte R. Metabolomic differentiation of Cannabis sativa cultivars using ${ }^{1} \mathrm{H}$ NMR spectroscopy and principal component analysis. J Nat Prod 2004; 67: 953-957

16 Choi YH, Tapias EC, Kim HK, Lefeber AWM, Erkelens C, Verhoeven JTJ, Brzin J, Zel J, Verpoorte R. Metabolic discrimination of Catharanthus roseus leaves infected by phytoplasma using ${ }^{1} \mathrm{H}-\mathrm{NMR}$ spectroscopy and multivariate data analysis. Plant Physiol 2004; 135: 2398-2410

17 Choi YH, Sertic S, Kim HK, Wilson EG, Michopoulos F, Lefeber AWM, Erkelens $C$, Kricun SDP, Verpoorte R. Classification of Ilex species based on metabolomic fingerprinting using NMR and multivariate data analysis. J Agric Food Chem 2005; 53: 1237-1245

18 Suhartono L, Van Iren F, De Winter W, Roytrakul S, Choi YH, Verpoorte R. Metabolic comparison of cryopreserved and normal cells from Tabernaemontana divaricata suspension cultures. Plant Cell Tissue Org Cult 2005; 83: 59-66

19 Kim HK, Choi YH, Erkelens C, Lefeber AWM, Verpoorte R. Metabolic fingerprinting of Ephedra species using ${ }^{1} \mathrm{H}-\mathrm{NMR}$ spectroscopy and principal component analysis. Chem Pharm Bull 2005; 53: 105-109 
20 Hendrawati O, Yao Q Kim HK, Linthorst HJM, Erkelens C, Lefeber AWM Choi YH, Verpoorte R. Metabolic differentiation of Arabidopsis treated with methyl jasmonate using nuclear magnetic resonance spectroscopy. Plant Sci 2006; 170: 1118-1124

21 Liang Y-S, Choi YH, Kim HK, Linthorst HJM, Verpoorte R. Metabolomic analysis of methyl jasmonate treated Brassica rapa leaves by two dimensional NMR spectroscopy and multivariate analysis. Phytochemistry 2006; 67: 2503-2511

22 Widarto HT, Van der Meijden E, Lefeber AWM, Erkelens C, Kim HK, Choi $Y H$, Verpoorte R. Metabolomic differentiation of Brassica rapa leaves attacked by herbivore using two dimensional nuclear magnetic resonance spectroscopy. J Chem Ecol 2006; 32: 2417-2428

23 Le Gall G, Colquhoun IJ, Defernez M. Metabolite profiling using ${ }^{1} \mathrm{H}$ NMR spectroscopy for quality assessment of green tea, Camellia sinensis (L.). J Agric Food Chem 2004; 52: 692-700

24 Ward JL, Harris C, Lewis J, Beale MH. Assessment of ${ }^{1} \mathrm{H}-\mathrm{NMR}$ spectroscopy and multivariate analysis as a technique for metabolite fingerprinting of Arabidopsis thaliana. Phytochemistry 2003; 62: 949-957

25 Frédérich $M$, Choi $Y H$, Verpoorte R. Quantitative analysis of strychnine and brucine in Strychnos nux-vomica using ${ }^{1} \mathrm{H}-\mathrm{NMR}$. Planta Med 2003; 69: 1169-1171

26 Kruger NJ, Troncoso-Ponce AT, Ratcliffe RG. ${ }^{1} \mathrm{H}$ NMR metabolite fingerprinting and metabolomic analysis of perchloric acid extracts from plant tissues. Nat Protoc 2008; 3: 1001-1012

27 Rasmussen B, Cloarec O, Tang H, Staerk D, Jaroszewski J. Multivariate analysis of integrated and full-resolution ${ }^{1} \mathrm{H}-\mathrm{NMR}$ spectral data from complex pharmaceutical preparations: St. John's wort. Planta Med 2006; 72: 556-563

28 Choi YH, Choi H-K, Hazekamp A, Bermejo P, Schilder YDC, Erkelens C, Verpoorte R. Quantitative analysis of bilobalide and ginkgolides from Ginkgo biloba leaves and ginkgo products using ${ }^{1} \mathrm{H}-\mathrm{NMR}$. Chem Pharm Bull 2003; 51: 158-161

29 Kim HK, Choi YH, Chang W-T, Verpoorte R. Quantitative analysis of ephedrine analogues from Ephedra species using ${ }^{1} \mathrm{H}-\mathrm{NMR}$. Chem Pharm Bull 2003; 51: 1382-1385

30 Schripsema J, Erkelens C, Verpoorte R. Intra-and extracellular carbohydrates in plant cell cultures investiated by ${ }^{1} \mathrm{H}-\mathrm{NMR}$. Plant Cell Rep 1991; 9: 527-530

31 Beckwith-Hall BM, Thompson NA, Nicholson JK, Lindon J, Holmes E. A metabonomic investigation of hepatotoxicity using diffusion-edited ${ }^{1} \mathrm{H}$-NMR spectroscopy of blood serum. Analyst 2003; 128: 814-818

32 Phalaraksh C, Lenz EM, Lindon JC, Nocholson JK, Farrant RD, Reynolds SE, Wilson ID, Osborn D, Weeks JM. NMR spectroscopic studies on the hemolymph of the tobacco hornworm, Manduca sexta: assignment of ${ }^{1} \mathrm{H}$ and ${ }^{13} \mathrm{C}$ NMR spectra. Insect Biochem Mol Biol 1999; 29: 795-805

33 Davis RA, Charlton AJ, Godward J, Jones SA, Harrison M, Wilson JC. Adaptive binning: an improved binning method for metabolomics data using the undecimated wavelet transform. Chemometr Intell Lab Syst 2007; 85: 144-154

$34 \mathrm{Kim}$ HK, Choi YH, Verpoorte R. Metabolomic analysis of Catharanthus roseus using NMR and principal component analysis. In: Saito K, Dixon RA, Willmitzer L, editors. Biotechnology in agriculture and forestry 57 plant metabolomics. Leipzig: Springer; 2006: 261-276

35 Defernez M, Colquhoun IJ. Factors affecting the robustness of metabolite fingerprinting using ${ }^{1} \mathrm{H}$ NMR spectra. Phytochemistry 2003; 62: 1009 1017

36 Forshed J, Schuppe-Koistinen I, Jacobsson SP. Peak alignment of NMR signals by means of a genetic algorithm. Anal Chim Acta 2003; 487: 189199

37 Lee G-C, Woodruff DL. Beam search for peak alignment of NMR signals. Anal Chim Acta 2004; 513: 413-416

38 Eriksson L, Johansson E, Kettaneh-Wold N, Wold S. Multi- and megabariate data analysis. Principles and applications. Umeå: Umetrics; 2001

39 Berger S, Braun S. 200 and more NMR experiments. Weinheim: WileyVCH; 2004

40 Kemsley EK. Discriminant analysis and class modelling of spectroscopic data. Chichester: John Wiley; 1998

41 Lindon JC, Holmes E, Nicholson JK. Pattern recognition methods and applications in biomedical magnetic resonance. Prog Nucl Magn Reson Spectrosc 2001; 39: 1-40

42 Colquhoun IJ. Use of NMR for metabolic profilind in plant systems. J Pestic Sci 2007; 32: 200-212

43 Keun HC, Ebbels TMD, Antti H, Bollard ME, Beckonert O, Holmes E Lindon $J C$, Nicholson JK. Improved analysis of multivariate data by variable sta- bility scaling: application to NMR-based metabonomic profiling. Anal Chim Acta 2003; 490: 265-276

44 Viant MR. Improved methods for the acquisition and interpretation of NMR metabolomic data. Biochem Biophys Res Commun 2003; 310: 943-948

45 Wolters AM, Jayawickrama DA, Sweedler JV. Microscale NMR. Curr Opin Chem Biol 2002; 6: 711-716

46 Viel S, Capitani D, Mannina L, Segre A. Diffusion-ordered NMR spectroscopy: a versatile tool for the molecular weight determination of uncharged polysaccharides. Biomacromolecules 2003; 4: 1843-1847

47 Ambrus A, Friedrich K, Somogyi Á. Oligomerization of nitrophorins. Anal Biochem 2006; 352: 286-295

48 Politi M, Groves P, Chávex I, Canada FJ, Jiménez-Barbero J. Useful application of DOSY experiments for the study of mushroom polysaccharides. Carbohydr Res 2006; 341: 84-89

49 Fraceto LF, Oyama Jr S, Nakaie CR, Spisni A, De Paula E, Pertinhez TA. Interaction of local anesthetics with a peptide encompassing the IV/ SA-S5 inler of the $\mathrm{Na}^{+}$channel. Biophys Chem 2006; 123: 29-39

50 Zeng B, Zhu X, Cai S, Chen Z. Interactions between diperoxovanadate complex and amide ligands in aqueous solution. Spectrochim Acta Part A 2007; 67: 202-207

51 Kapur GS, Findeisen M, Berger S. Analysis of hydrocarbon mixtures by diffusion-ordered NMR spectroscopy. Fuel 2000; 79: 1347-1351

52 Rauter AP, Martins A, Borges C, Ferreira J, Justino J, Bronze M-R, Coelho $A V$, Choi $Y H$, Verpoorte $R$. Liquid chromatography-diode array detection-electrospray ionisation mass spectrometry/nuclear magnetic resonance analyses of the anti-hyperglycemic flavonoid extract of Genista tenera: structure elucidation of a flavonoid-C-glycoside. J Chromatogr A 2005; 1089: 59-64

53 Bradley SA, Krishnamurthy $\mathrm{K}, \mathrm{Hu} \mathrm{H}$. Simplifying DOSY spectra with selective TOCSY edited preparation. J Magn Reson 2005; 172: 110-117

54 Lucas LH, Otto W, Larive CK. The 2D-J-DOSY experiment: resolving diffusion coefficients in mixtures. J Magn Reson 2002; 156: 138-145

55 Barjat A, Morris GA, Swanson AG. A three-dimensional DOSY-HMQC experiment for the high-resolution analysis of complex mixtures. J Magn Reson 1998; 131: 131-138

56 Wang Y, Tang H, Nicholson JK, Hylands PJ, Sampson J, Whitcombe S, Stewart CG, Caiger S, Oru I, Holmes E. Metabolomic strategy for the classification and quality control of phytomedicine: a case study of chamomile flower (Matricaria recutita L.). Planta Med 2004; 70: 250-255

57 Frédérich $M$, Choi $Y H$, Angenot L, Harnischfeger G, Lefeber AWM, Verpoorte R. Metabolomic analysis of Strychnos nux-vomica, icaja and ignatii extracts by ${ }^{1} \mathrm{H}$ nuclear magnetic resonance spectrometry and multivariate analysis techniques. Phytochemistry 2004; 65: 19932001

58 Yang SY, Kim HK, Lefeber AWM, Erkelens C, Angelova N, Choi YH, Verpoorte $R$. Application of two-dimensional nuclear magnetic resonance spectroscopy to quality control of ginseng commercial products. Planta Med 2006; 72: 364-369

59 Shin YS, Bang KH, In DS, Kim OT, Hyun DY, Ahn IO, Ku BC, Kim SW, Seong NS, Cha SW, Lee D, Choi HK. Fingerprinting analysis of fresh ginseng roots of different ages using H-NMR spectroscopy and principal component analysis. Arch Pharm Res 2007; 30: 1625-1628

60 Kang J, Lee S, Kang S, Kwon HN, Park JH, Kwon SW, Park S. NMR-based metabolomics approach for the differentiation of ginseng (Panax ginseng) roots from different origins. Arch Pharm Res 2008; 31: 330-336

61 Tarachiwin L, Katoh A, Ute K, Fukusaki E. Quality evaluation of Angelica acutiloba Kitagawa roots by ${ }^{1} \mathrm{H}$ NMR-based metabolic fingerprinting. J Pharm Biomed Anal Advance online publication 2008; DOI: 10.1016/ j.jpba

62 Van der Kooy F, Verpoorte R, Marion Meyer JJ. Metabolomic quality control of claimed anti-malarial Artemisia afra herbal remedy and A. afra and A. annua plant extracts. South African J Bot 2008; 74: 186-189

63 Bailey NIC, Sampson J, Hylands PJ, Nicholson JK, Holmes E. Multi component metabolic classification of commercial feverfew preparations via high-filed ${ }^{1} \mathrm{H}-\mathrm{NMR}$ spectroscopy and chemometrics. Planta Med 2002; 68: 734-738

64 Bilia AR, Bergonzi MC, Lazari D, Vincieri FF. Characterization of commercial kava-kava herbal drug and herbal drug preparations by means of nuclear magnetic resonance spectroscopy. J Agric Food Chem 2002; 50: 5016-5025

65 Bailey NJC, Wang YL, Sampson J, Davis W, Whitcombe I, Hylands PJ, Croft SL, Holmes E. Prediction of anti-plasmoidal activity of Artemisia annua extracts: application of ${ }^{1} \mathrm{H}-\mathrm{NMR}$ spectroscopy and chemometrics. J Pharm Biomed Anal 2004; 35: 117-126 
66 Roos G, Röseler C, Berger-BüterK, Simmen U. Classification and correction of St. John's wort extracts by nuclear magnetic resonance spectroscopy, multivariate data analysis and pharmacological activity. Planta Med 2004; 70: 771-777

67 Holmes E, Tang $H$, Wang $Y$, Seger $C$. The assessment of plant metabolite profiles by NMR-based methodologies. Planta Med 2007; 72: 771-785

68 Bedair M, Sumner LW. Current and emerging mass-spectrometry technologies for metabolomics. Trends Anal Chem 2008; 27: 238-250

69 García-Villalba R, León C, Dinelli G, Segura-Carretero A, Fernández-Gutiérrez A, Garcia-Cañas V, Cifuentes A. Comparative metabolomic study of transgenic versus conventional soybean using capillary electrophoresis-time-of-flight mass spectrometry. J Chromatogr A 2008; 1195: 164-173

70 Harris CS, Burt AJ, Saleem A, Le PM, Martineau LC, Haddad, PS, Bennett SAL, Arnason JT. A single HPLC-PAD-APCI/MS method for the quantitative comparison of phenolic compounds found in leaf, stem, root and fruit extracts of Vaccinium angustifolium. Phytochem Anal 2007; 18: 161-169

71 Grata E, Boccard J, Glauser G, Carrupt P-A, Farmer EE, Wolfender J-L, Rudaz $S$. Development of a two-step screening ESI-TOF-MS method for rapid determination of significant stress-induced metabolome modifications in plant leaf extracts: the wound response in Arabidopsis thaliana as a case study. J Sep Sci 2007; 30: 2268-2278

72 Vorst O, De Vos CHR, Lommen A, Staps RV, Visser RGF, Bino RJ, Hall RD. A non-directed approach to the differential analysis of multiple LC-MSderived metabolic profiles. Metabolomics 2005; 1: 169-180

73 De Vos RCH, Moco S, Lommen A, Keurentjes JJB, Bino RJ, Hall RD. Untargeted large-scale plant metabolomics using liquid chromatography coupled to mass spectrometry. Nat Protoc 2007; 2: 778-791

74 Zeng ZD, Liang YZ, Chau FT, Chen S, Daniel MKW, Chan CO. Mass spectral profiling: an effective tool for quality control of herbal medicines. Anal Chim Acta 2007; 604: 89-98

75 Pongsuwan W, Bamba T, Yonetani T, Kobayashi A, Fukusaki E. Quality prediction of Japanese green tea using pyrolyzer coupled GC/MS based metabolic fingerprinting. J Agric Food Chem 2008; 56: 744-750

76 Xie G, Plumb R, Su M, Xu Z, Zhao A, Qiu M, Long X, Liu Z, Jia W. Ultra-performance LC/TOF MS analysis of medicinal Panax herbs for metabolomic research. J Sep Sci 2008; 31: 1015-1026

77 Williams BJ, Cameron CJ, Workman R, Broeckling CD, Sumner LW, Smith JT. Amino acid profiling in plant cell cultures: an inter-laboratory comparison of CE-MS and GC-MS. Electrophoresis 2007; 28: 1371-1379

78 Timischl B, Dettmer K, Kaspar H, Thieme M, Oefner PJ. Development of a quantitative, validated capillary electrophoresis-time of flight-mass spectrometry method with integrated high-confidence analyte identification for metabolomics. Electrophoresis 2008; 29: 2203-2214

79 Inbaraj BS, Lua $H$, Hung CF, Wu WB, Lin CL, Chen BH. Determination of carotenoids and their esters in fruits of Lycium barbarum Linnaeus by HPLC-DAD-APCI-MS. J Pharm Biomed Anal 2008; 47: 812-818

80 Thiocone A, Farmer EE, Wolfender J-L. Screening for wound-induced oxylipins in Arabidopsis thaliana by differential HPLC-APCI/MS profiling of crude leaf extracts and subsequent characterisation by capillary-scale NMR. Phytochem Anal 2008; 19: 198-205

81 Clarkson C, Strk D, Hansen SH, Smith PJ, Jaroszewski JW. Identification of major and minor constituents of Harpagophytum procumbens (Devil's Claw) using HPLC-SPE-NMR and HPLC-ESIMS/APCIMS. J Nat Prod 2006; 69: $1280-1288$

82 Aharoni A, De Vos CHR, Verhoeven HA, Maliepaard CA, Kruppa G, Bino R, Goodenowe $D B$. Nontargeted metabolome analysis by use of Fourier transform ion cyclotron mass spectrometry. OMICS 2002; 6: 217-234

83 Ohta D, Shibata D, Kanaya S. Metabolic profiling using Fourier-transform ion-cyclotron-resonance mass spectrometry. Anal Bioanal Chem 2007; 389: 1469-1475

84 Kang J, Zhou L, Sun J, Han J, Guo D-A. Chromatographic fingerprint analysis and characterization of furocoumarins in the roots of Angelica dahurica by HPLC/DAD/ESI-MSn technique. J Pharm Biomed Anal 2008; 47: 778-785

85 Thysell E, Pohjanen E, Lindberg J, Schuppe-Koistinen I, Moritz T, Jonsson P, Antti $H$. Reliable profile detection in comparative metabolomics. OMICS 2007; 11: 209-224

86 Broeckling CD, Reddy IR, Duran AL, Zhao X, Sumner LW. MET-IDEA: data extraction tool for mass spectrometry-based metabolomics. Anal Chem 2006; 78: 4334-4341
87 Su J, Fu P, Shen Y, Zhang C, Liang M, Liu R, Li H, Zhang W. Simultaneous analysis of flavonoids from Hypericum japonicum Thunb. ex Murray (Hypericaceae) by HPLC-DAD-ESI/MS. J Pharm Biomed Anal 2008; 46: $342-348$

88 Liu Q Li H, Hang T. Identification of chemical components illegally mixed in traditional Chinese medicine preparations. Zhongguo Yaoshi 2007; 10: 735-737

$89 \mathrm{Li} \mathrm{Y,} \mathrm{Hu} \mathrm{Z,} \mathrm{He} \mathrm{L.} \mathrm{An} \mathrm{approach} \mathrm{to} \mathrm{develop} \mathrm{binary} \mathrm{chromatographic} \mathrm{fin-}$ gerprints of the total alkaloids from Caulophyllum robustum by high performance liquid chromatography/diode array detector and gas chromatography/mass spectrometry. J Pharm Biomed Anal 2007; 43: 1667-1672

90 Jensen AG, Ndjoko K, Wolfender J-L, Hostettmann K, Camponovo F, Soldati $F$. Liquid chromatography-atmospheric pressure chemical ionisation/mass spectrometry: a rapid and selective method for the quantitative determination of ginkgolides and bilobalide in ginkgo leaf extracts and phytopharmaceuticals. Phytochem Anal 2002; 13: 31-38

91 Tiberti LA, Yariwake JH, Ndjoko K, Hostettmann K. Identification of flavonols in leaves of Maytenus ilicifolia and M. aquifolium (Celastraceae) by LC/UV/MS analysis. J Chromatogr B 2007; 846: 378-384

92 Bruni R, Pellati F, Bellardi MG, Benvenuti S, Paltrinieri S, Bertaccini A, Bianchiet A. Herbal drug quality and phytochemical composition of Hypericum perforatum L. affected by ash yellows phytoplasma infection. J Agric Food Chem 2005; 53: 964-968

93 Kovacs H, Moskau D, Spraul M. Cryogenically cooled probes - a leap in NMR technology. Prog Nucl Magn Reson Spectrosc 2005; 46: 131-155

94 Schroeder FC, Gronquist M. Extending the scope of NMR spectroscopy with microcoil probes. Angew Chem Int Ed Engl 2006; 45: 7122-7131

95 Kopka JJ. Current challenges and developments in GC-MS based metabolite profiling technology. Biotechnology 2006; 124: 312-322

96 Exarchou V, Godejohann M, van Beek TA, Gerothanassis IP, Vervoort J. LC-UV-solid-phase extraction-NMR-MS combined with a cryogenic flow probe and its application to the identification of compounds present in Greek oregano. Anal Chem 2003; 75: 6288-6294

97 Seger C, Godejohann M, Tseng LH, Spraul M, Girtler A, Sturm S, Stuppner $H$. LC-DAD-MS/SPE-NMR hyphenation. A tool for the analysis of pharmaceutically used plant extracts: identification of isobaric iridoid glycoside regioisomers from Harpagophytum procumbens. Anal Chem 2005; 77: 878-885

98 Wolfender JL, Rodrigues S, Hostettmann $K$, Hiller W. Liquid chromatography/ultraviolet/mass spectrometric and liquid chromatography/ nuclear magnetic resonance spectroscopic analysis of crude extract of Gentianaceae species. Phytochem Anal 1997; 8: 97-104

99 Ioset JR, Wolfender JL, Marston A, Gupta MP, Hostettmann K. Identification of two isomeric meroterpenoid naphtoquinones from Cordia linnaei by liquid chromatography-mass spectrometry and liquid chromatography-nuclear magnetic resonance spectroscopy. Phytochem Anal 1999; 10: 137-142

100 Queiroz EF, Wolfender JL, Atindehou KK, Traore D, Hostettmann K. Online identification of the antifungal constituents of Erythrina vogelii by liquid chromatography with tandem mass spectrometry, ultraviolet adsorbance detection and nuclear magnetic resonance spectrometry combined with chroamtographic micro-fractionation. J Chromatogr A 2002; 974: 123-134

101 Cogne AL, Queiroz EF, Wolfender JL, Marston A, Mavi S, Hostettmann K. On-line identification of unstable catalpol derivatives from Jamesbrittenia fodina by LC-MS and LC-NMR. Phytochem Anal 2003; 14: 67-73

102 Ramm M, Wolfender JL, Queiroz EF, Hostettmann K, Hamburger M. Rapid analysis of nucleotide-activated sugars by high-performance liquid chromatography coupled with diode-array detection, electrospray ionization mass spectrometry and nuclear magnetic resonance. J Chromatogr A 2004; 1034: 139-148

103 Jaroszewski JW. Hyphenated NMR methods in natural products research, Part 1: direct hyphenation. Planta Med 2005; 71: 691-700

104 Glauser G, Guillarme D, Grata E, Boccard J, Thiocone A, Carrupt PA, Veuthey J-L, Rudaz S, Wolfender J-L. Optimized liquid chromatographymass spectrometry approach for the isolation of minor stress biomarkers in plant extracts and their identification by capillary nuclear magnetic resonance. J Chromatogr A 2008; 1180: 90-98

105 Grata E, Boccard J, Guillarme D, Glauser G, Carrupt PA, Farmer EE, Wolfender J-L, Rudaz S. UPLC-TOF-MS for plant metabolomics: a sequential approach for wound marker analysis in Arabidopsis thaliana. J Chromatogr B 2008; 871: 261-270

106 Swartz ME. UPLC ${ }^{\mathrm{TM}}$ : an introduction and review. J Liq Chromatogr Rel Technol 2005; 28: 1253-1263 
107 Nguyen DTT, Guillarme D, Rudaz S, Veuthey JL. Fast analysis in liquid chromatography using small particle size and high pressure. J Sep Sci 2006; 29: 1836-1848

108 Guan J, Laia CM, Li SP. A rapid method for the simultaneous determination of 11 saponins in Panax notoginseng using ultra performance liquid chromatography. J Pharm Biomed Anal 2007; 44: 996-1000

109 Li P, Xu G, Li SP, Wang YT, Fan TP, Zhao QS, Zhang QW. Optimizing ultraperformance liquid chromatographic analysis of 10 diterpenoid compounds in Salvia miltiorrhiza using central composite design. J Agric Food Chem 2008; 56: 1164-1171

110 Chen XJ, Ji H, Zhang QW, Tu PF, Wang YT, Guo BL, Li SP. A rapid method for simultaneous determination of 15 flavonoids in Epimedium using pressurized liquid extraction and ultra-performance liquid chromatography. J Pharm Biomed Anal 2008; 46: 226-235

111 Johnson KA, Plumb RJ. Investigating the human metabolism of acetaminophen using UPLC and exact mass oa-TOF MS. J Pharm Biomed Anal 2005; 39: 805-810

112 Wilson ID, Nicholson JK, Castro-Perez J, Granger JH, Johnson KA, Smith $B W$, Plumb RS. High resolution "ultra performance" liquid chromatography coupled to oa-TOF mass spectrometry as a tool for differential metabolic pathway profiling in functional genomic studies. J Proteome Res 2005; 4: 591-598

113 Nordström A, O'Maille G, Qin C, Siuzdak G. Nonlinear data alignment for UPLC-MS and HPLC-MS based metabolomics: quantitative analysis of endogenous and exogenous metabolites in human serum. Anal Chem 2006; 78: 3289-3295

114 Yu K, Little D, Plumb R, Smith B. High-throughput quantification for a drug mixture in rat plasma: a comparison of ultra performance liquid chromatography/tandem mass spectrometry with high-performance liquid chromatography/tandem mass spectrometry. Rapid Commun Mass Spectrom 2006; 20: 544-552

115 Jacob SS, Smith NW, Legido-Quigley C. Assessment of Chinese medicinal herb metabolite profiles by UPLC-MS-based methodology for the detection of aristolochic acids. J Sep Sci 2007; 30: 1200-1206

116 Naidong $W$. Bioanalytical liquid chromatography tandem mass spectrometry methods on underivatized silica columns with aqueous/organic mobile phases. J Chromatogr B 2003; 796: 209-224

117 Tolstikov VV, Fiehn O. Analysis of highly polar compounds of plant origin: combination of hydrophilic interaction chromatography and electrospray ion trap mass spectrometry. Anal Biochem 2002; 301: 298-307

118 Kind T, Tolstikov VV, Fiehn O, Weiss RH. A comprehensive urinary metabolomic approach for identifying kidney cancer. Anal Biochem 2007; 363: $185-195$

119 Dunn BW. Current trends and future requirements for the mass spectrometric investigation of microbial, mammalian and plant metabolomes. Physiol Biol 2008; 5: 1-24
120 Washburn M, Wolters D, Yates J. Large-scale analysis of the yeast proteome by multidimensional protein identification technology. Nat Biotechnol 2001; 19: 242-247

121 Wolters D, Washburn M, Yates J. An automated multidimensional protein identification technology for shotgun proteomics. Anal Chem 2001; 73: 5683-5690

122 Dugo P, Kumm T, Crupi ML, Cotroneo A, Mondello L. Comprehensive two-dimensional liquid chromatography combined with mass spectrometric detection in the analyses of triacylglycerols in natural lipidic matrixes. J Chromatogr A 2006; 1112: 269-275

123 Ryan D, Robards K. Analytical chemistry considerations in plant metabolomics. Sep Purif Rev 2006; 35: 319-356

124 Mondello L, Tranchida PQ Dugo P, Dugo G. Comprehensive two-dimensional gas chromatography-mass spectrometry: a review. Mass Spectrom Rev 2008; 27: 101-124

125 Shellie RA, Marriott PJ. Comprehensive two-dimensional gas chromatography-mass spectrometry analysis of Pelargonium graveolens essential oil using rapid scanning quadrupole mass spectrometry. Analyst 2002; 128: 879-883

126 Shellie RA, Marriot PJ, Huie CW. Comprehensive two-dimensional gas chromatography (GC'GC) and GC'GC-quadrupole MS analysis of Asian and American ginseng. J Sep Sci 2003; 26: 1185-1192

127 Shellie RA, Welthagen W, Zrostlikova J, Spranger J, Ristow M, Fiehn O, Zimmermann $R$. Statistical methods for comparing comprehensive two-dimensional gas chromatography-time-of-flight mass spectrometry results: metabolomics analysis of mouse tissue extracts. J Chromatogr A 2005; 1086: 83-90

128 Hope JL, Prazen BJ, Nilsson EJ, Jack RM, Synovec RE. Comprehensive two-dimensional gas chromatography with time-of-flight mass spectrometry detection: analysis of amino acid and organic acid trimethylsilyl derivatives, with application to the analysis of metabolites in rye grass samples. Talanta 2005; 65: 380-388

129 Pierce KM, Hope JL, Hoggard JC, Synovec RE. Principal component analysis based method to discover chemical differences in comprehensive two-dimensional gas chromatography with time-of-flight mass spectrometry (GCXGC-TOFMS) separations of metabolites in plant samples. Talanta 2006; 70: 797-804

130 Verpoorte R, Choi YH, Mustafa NR, Kim HK. Metabolomics: back to basics. Phytochem Rev 2008; 7: 525-537

131 Schad M, Mungur R, Fiehn O, Kehr J. Metabolic profiling of laser microdissected vascular bundles of Arabidopsis thaliana. Plant Methods 2005; $1: 1-10$

132 Schneider B, Hölscher D. Laser microdissection and cryogenic nuclear magnetic resonance spectroscopy: an alliance for cell type-specific metabolite profiling. Planta 2007; 225: 763-770

133 Li SH, Schneider B, Gershenzon J. Microchemical analysis of laser-microdissected stone cells of Norway spruce by cryogenic nuclear magnetic resonance spectroscopy. Planta 2007; 225: 771-779 